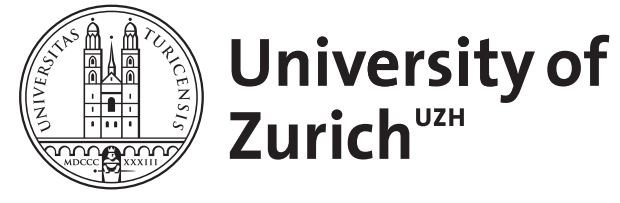

\title{
Neurobiological mechanisms of acute vertigo
}

\author{
Tarnutzer, A A ; Palla, A
}

\begin{abstract}
The vestibular system provides us with reflexive responses of eye movements and balance control, as well as with perceptual estimates of self-motion and gravity direction. Crucial to its proper functioning is a bilaterally balanced vestibular signal originating from the vestibular end organs in the inner ears and projecting via vestibular nerve afferents to the brainstem vestibular nuclei. Disturbances of the bilateral vestibular interplay become evident in cases of acute unilateral peripheral vestibular deafferentation. The resultant sudden imbalance of vestibular afferent tone at the level of the vestibular nuclei leads to pronounced ocular-motor and postural impairment, as well as to intensive vertigo and/or dizziness, accompanied by autonomic symptoms, such as nausea and vomiting. Subsequent compensatory mechanisms efficiently diminish these static symptoms (such as spontaneous nystagmus) within days and allow functional recovery of dynamic symptoms (such as blurred vision during fast head turns) to such a degree that most patients return to their normal daily activities within weeks. This article aims to provide an understanding about the pathophysiological changes after unilateral vestibular deafferentation and the current knowledge on the compensatory mechanisms.
\end{abstract}

DOI: https://doi.org/10.2217/fnl.13.17

Posted at the Zurich Open Repository and Archive, University of Zurich

ZORA URL: https://doi.org/10.5167/uzh-85003

Journal Article

Accepted Version

Originally published at:

Tarnutzer, A A; Palla, A (2013). Neurobiological mechanisms of acute vertigo. Future Neurology, $8(4): 445-455$.

DOI: https://doi.org/10.2217/fnl.13.17 


\section{Neurobiological Mechanisms of Acute Vertigo}

Alexander A. Tarnutzer and Antonella Palla

Department of Neurology, University Hospital Zurich, Zurich, Switzerland

Corresponding author:

A. A. Tarnutzer, M.D.

Department of Neurology

University Hospital Zurich

Frauenklinikstrasse 26

CH-8091 Zurich

Switzerland

Phone: +41-44-255-1111 Fax: +41-44-255-4380

Email: alexander.tarnutzer@access.uzh.ch

\section{$\underline{\text { Key words: }}$}

vestibular system, acute vestibular deafferentation, vestibular compensation, sensory substitution, imaging.

\section{Grant / financial support:}

Swiss National Science Foundation (grant number: 32003B_130163/1), Berne, Switzerland; the Betty and David Koetser Foundation for Brain Research, Zurich, Switzerland; Center of Integrative Human Physiology, University of Zurich, Switzerland; Bonizzi-ThelerFoundation, Zurich, Switzerland; Baasch Medicus Foundation; Zurich Center for Integrative Human Physiology

Disclosure:

The authors have nothing to disclose. 
Abbreviations

MVN: medial vestibular nucleus

LVN: lateral vestibular nucleus

SCC: semicircular canal

UVD: unilateral vestibular deafferentation

VC: vestibular compensation

VN: vestibular nucleus

VOR: vestibulo-ocular reflex

VSR: vestibulo-spinal reflex 


\section{Summary}

The vestibular system provides us with reflexive responses of eye movement and balance control as well as with perceptual estimates of self-motion and gravity direction. Crucial to its proper functioning is a bilaterally balanced vestibular signal originating from the vestibular end organs in the inner ears and projecting via vestibular nerve afferents to the brainstem vestibular nuclei. Disturbances of the bilateral vestibular interplay become evident in case of an acute unilateral peripheral vestibular deafferentation. The resultant sudden imbalance of vestibular afferent tone at the level of the vestibular nuclei leads to pronounced ocular motor and postural impairment as well as to intensive vertigo and / or dizziness, accompanied by autonomic symptoms such as nausea and vomiting. Subsequent compensatory mechanisms efficiently diminish these static symptoms (such as spontaneous nystagmus) within days and allow functional recovery of dynamic symptoms (such as blurred vision during fast head turns) to such a degree that most patients return to their normal daily activities within weeks. This review aims to give an understating about the pathophysiologic changes after unilateral vestibular deafferentation and the current knowledge on the compensatory mechanisms. 


\section{Basic principles of the vestibular system}

A prerequisite for normal functioning of the vestibular system is a bilaterally balanced discharge rate at the level of the vestibular nuclei. Sensory signals originating from the vestibular end organs (i.e., the semicircular canals (SCC) and the macular organs) in the inner ears are projected via vestibular nerve afferents to the vestibular nuclei (VN) neurons located in the brainstem. Bilateral pathways further transmit the vestibular signals from the VN to the brainstem ocular-motor, spinal-motor and cerebellar neurons for reflexive responses and to the thalamus and higher-cortical areas for movement sensation and gravity estimation [1]. The significance of the balanced interplay becomes evident in the occurrence of a sudden unilateral vestibular deafferentation (UVD). In fact, damage to the vestibular labyrinth (e.g. secondary to ischemia, inflammation, endolymphatic hydrops or labyrinthectomy) or to the vestibular nerve (e.g. secondary to ischemia, inflammation or neurectomy) results in a clinical condition termed acute vestibular syndrome and manifests as prolonged vertigo and / or dizziness accompanied by nausea and / or vomiting, head-motion intolerance, spontaneous nystagmus and gait imbalance [2]. In face of the intensity of complaints, their disappearance within days to weeks is a stunning example of profound behavioral and functional recovery. Moreover, since regeneration after a complete destruction of the vestibular labyrinth or the vestibular nerve is lacking [3], recovery must be attributed to central neuronal plasticity. Therefore, UVD may serve as an attractive model to study deafferentation-induced plasticity, i.e. vestibular compensation $(\mathrm{VC})[4,5]$.

In the following sections we will first address the physiological properties of the vestibular system, followed by the pathophysiological changes related to UVD. Subsequently, we will summarize the current knowledge of $\mathrm{VC}$ and address its impact on treatment strategies. 


\subsection{The three-neuron vestibular reflex pathway}

\subsubsection{Anatomical organization}

The brain interprets imbalances, i.e. asymmetries, in vestibular input due to an acute pathologic process in the same way that it interprets imbalances due to a physiological stimulus. Understanding the physiological processes underlying motion is therefore a prerequisite for understanding vestibular disorders [6].

The classical "three-neuron arc" originates in the vestibular end organs and projects via vestibular nerve afferents to the $\mathrm{VN}$ and further via second-order vestibular nuclei neurons to extra-ocular motor neurons, forming the vestibulo-ocular reflex (VOR) for gaze control, and to spinal-motor neurons, forming vestibulo-spinal reflexes (VSR) for balance control. When the head is upright and at rest, vestibular end organ cells as well as vestibular afferents and VN cells have a constant spontaneous (i.e. tonic) firing rate, which is roughly equal on both sides. When the head then rotates towards one side, e.g. the right, there is an increase of vestibular neuron activity of that side, i.e. the right, and a decrease of activity on the contralateral side, i.e. the left. This change of firing rate, resulting in an imbalance between both sides, indicates the brain that a head movement occurred. As similar imbalance occurs with a unilateral vestibular impairment, it is evident that vertigo is the resultant of the brain signaling an ongoing movement. Details on the functional organization of the vestibular end organs are provided in figure 1.

\subsubsection{Electrophysiological properties}

The vestibular afferents are functionally grouped on the basis of the regularity of their resting discharge rate. Regular afferents have a low sensitivity to head rotations and terminate onto Type II hair cells. Irregular afferents, in contrast, terminate onto both Type I and Type II 
hair cells and demonstrate both low (when terminating on Type I cells) and high (when terminating on Type II cells) sensitivity to head rotations. The same pattern of functionally distinct Type I and Type II cells observed in the vestibular afferents is continued within the VN neurons: two cell populations (Type I and Type II VN neurons) cause two complementary responses following the "push-pull principle": 1) ipsilateral regular firing Type I medial vestibular nucleus (MVN) neurons are excited via direct vestibular afferent input whereas 2) inhibitory ipsilateral irregular firing Type II MVN neurons are inhibited via commissural projections from the contralateral Type I MVN neurons [7] (see Fig. 2 for a schematic illustration of the "push-pull principle") .

While the semicircular canals are linked predominantly to the MVN, the primary otolith afferents project mainly to the lateral vestibular nucleus (LVN). Connections from the LVN to the spinal cord participate in postural control via vestibulo-spinal reflexes. Unlike the MVN, reciprocal commissural connections between the LVN are lacking [7].

\subsubsection{Neurochemistry}

Four major neurotransmitters, which are further modulated by other neurotransmitters, are known to be involved in the three-neuron vestibular reflex pathway (see [8] for a review). Glutamate is the major excitatory [9] and acetylcholine the primary inhibitory neurotransmitter [10] of the peripheral and central vestibular system. Gamma-aminobutyric acid (GABA) and glycine are inhibitory neurotransmitters found predominantly in connections between second order vestibular neurons and the ocular motor neurons [11]. Regarding GABA, two types of receptors, GABA-A and GABA-B, seem to play a pivotal role in the coordination of the central vestibular pathways (see [12] for a detailed review). Both receptors are expressed within the VN: while GABA-A likely mediates the commissural inhibition between the MVN, the cerebellar inhibition of the $\mathrm{VN}$ and the generation of 
spontaneous nystagmus, GABA-B function is less clear. A role in modulating commissural pathways and the vestibulo-ocular reflex is assumed.

The circuitry, by which several other neurotransmitters affect vestibular responses, is less well understood. Relevant for clinical purposes is, for example, histamine, as antihistamines modulate symptoms of motion sickness [13]. Histamine is found diffusely in central vestibular structures and both the $\mathrm{H} 1$ and $\mathrm{H} 2$ subtypes seem to affect vestibular responses [9]. Norepinephrine participates in modulating the intensity of central vertiginous reactions to motion stimulation [14] and also affects adaptation. Dopamine has been shown to alter VC mechanisms [15], and serotonin is involved in nausea [16].

\section{Pathophysiological changes occurring in unilateral vestibular deafferentation}

Immediately after UVD, a drop of normal resting discharge rate and sensitivity of ipsilesional Type I MVN neurons is observed [17-19], as the excitatory synaptic input from the ipsilateral vestibular afferents is lacking (see figure 2, panel B). In addition, there is also an increased inhibitory drive from the contralesional MVN and transmitted via the reciprocal commissural inhibitory system to ipsilesional Type I MVN neurons (see [20] for review). The firing rate of contralesional Type I MVN neurons, at the same time, either remains unchanged or increases slightly $[21,22]$, as the inhibitory drive of contralesional Type II MVN neurons (normally provided by ipsilesional Type I neurons) on these neurons is lost [17] (see also Figure 2).

The combination of lacking peripheral vestibular sensory input to the ipsilesional MVN, increased ipsilesional MVN inhibition by the contralesional MVN neurons and disinhibition of the contralesional MVN results in a large imbalance in the resting discharge rate between both sides. As pointed out by Bergquist and colleagues, MVN neurons are central in the generation of the vestibulo-ocular reflex and the vestibulo-spinal reflex [23, 24]. 
Accordingly, it is this marked asymmetry at the level of the MVN neurons that is believed to cause the acute ocular motor, postural and sensory deficits and complaints.

\subsection{Early vestibular compensation}

The goal of $\mathrm{VC}$ is to restore symmetrical vestibulo-ocular and vestibulo-spinal responses and to minimize vertigo. Recovery of spontaneous activity of the deafferented ipsilesional MVN is achieved, early on, by increasing its intrinsic neuronal excitability most likely by rapid changes in several neurotransmitters including GABA and glycine [18, 25, 26]. At the same time, the contralesional disinhibited MVN may regain normal discharge levels by disinhibition of the inhibitory commissural pathway [21]. These changes occur within the first hours after UVD, although the exact time line is species-dependent. In the guinea-pig, the resting discharge rate of the ipsilesional Type I neurons is restored in part after 52 to 60 hours [18]. This time line is in agreement with functional recovery of static symptoms in these animals. During the same interval, ipsilesional Type II neurons [18] and contralesional Type I and Type II neurons [21] return from the initially increased resting discharges rate to normal values, which may facilitate disinhibition and returning to a normal resting rate in ipsilesional Type I neurons.

Not only semicircular canal afferents are interrupted after UVD, but also otolith afferents projecting to the LVN. Compared to alterations in the MVN, neuronal changes in the ipsilesional LVN are distinct and also diverse within the LVN: while the number of neurons located in the rostroventral areas responding to otolith signals and their overall resting discharge rate decreases, the number of neurons and their sensitivity to otolith signals in the dorsocaudal part increases [27-29]. In the contralesional LVN the resting discharge rate is slightly reduced after UVD, resulting in an overall asymmetrical neuronal activity immediately after UVD [30, 31]. Compared to the ipsilesional MVN, recovery in ipsilesional 
LVN seems to be superior, which is in accordance with the observation that vestibulo-spinal reflexes are compensated better than vestibulo-ocular reflexes [7].

Taken together, within a few days ipsilesional resting discharge activities of both the MVN and the LVN recover [19], leading to a rebalance that approximately parallels the behavioral recovery of static deficits such as spontaneous nystagmus and postural imbalance $[32,33]$. In the following, we will review in more depth some of these compensatory strategies including biochemical modifications, changes in electrophysiological properties, cell proliferation and re-organization of synaptic connections.

\subsubsection{Biochemical mechanisms of recovery}

In search for pharmacological treatments of vestibular disorders, a number of studies have focused on molecular changes after UVD. Although it is uncontested that the four major neurotransmitters, GABA, glycine, glutamate, and acetylcholine, are involved in the process of neuroplastic changes in $\mathrm{VC}$, a clear picture has not yet emerged, probably because of differences within species. In rats, for example, Yamanaka [34] reported a compensatory down-regulation of inhibitory GABA-A and GABA-B receptors ipsilesional and an upregulation of GABA-B receptors contralesional. Changes in GABA receptor efficacy, thus, could be interpreted in the context of reducing the imbalance between the bilateral MVN [20, 34, 35]. No such changes, however, were found in mice within one week after UVD, while still in mice instead an increase of glutamate receptor activation in the MVN was observed as early as within 4 hours after UVD [36]. Similarly, while studies agree that glutaminergic $N$ methyl-D-aspartate (NMDA) receptors are involved in long-term potentiation and depression in the $\mathrm{VN}$, some studies have proposed as mechanism an up-regulation of NMDA receptors, but others have questioned this (see [37] for review). As to the putative role of acetylcholine in $\mathrm{VC}$, it is generally assumed that it contributes to ocular motor and postural control, as injection of acetylcholine in $\mathrm{VN}$ of squirrel monkeys, causes ocular motor and postural 
deficits (for review see [38]).

Levels of stress hormones and histamine influence vestibular plasticity mechanisms as well: labyrinthectomized rats demonstrated a beneficial effect of corticosteroids on vestibular plasticity when the animals were awake and experiencing the stress normally associated with deafferentation [39]. This suggests that the stress-related activation of glucocorticoid receptors also contributes to recovery after UVD. Histamine influences VC by modulatory actions on glycine and GABA release in the MVN. Specifically, the direct H3-receptormediated inhibition of GABA release is down-regulated for at least 3 weeks in both ipsilesional and contralesional MVNs after UVD in rats [40].

\subsubsection{Changes in intrinsic excitability}

In parallel to the biochemical changes, the intrinsic electrophysiological properties of the MVN neurons are also modulated (for a review see [41]). Specifically, the intrinsic excitability of ipsilesional MVN cells is increased after UVD [25], which was interpreted as a strategy to counteract the initial disfacilitation and the increased commissural inhibition from the contralesional MVN. Potential underlying mechanisms allowing such changes in MVN neuronal excitability are intracellular modulations of electrolyte levels including calcium and potassium [42], the down-regulation of inhibitory GABA [34] and glycine receptors [43] and the increased activation of glucocorticoid receptors [39]. Guilding and Dutia [44] applied a synaptic blockade of GABA, glycine and glutamate receptors in vitro to evaluate its impact on intrinsic excitability of deafferented MVN neurons in rats. They proposed that the increase in intrinsic excitability after UVD follows two stages: 1) in the very early stage, increases in excitability seem to be mediated by intrinsic cellular mechanisms (e.g. intracellular electrolyte modulation [42]), as synaptic blockade of GABA, glycine and glutamate receptors had no effect before 48 hours after UVD; 2) the maintenance of increased intrinsic excitability later on seems to depend on synaptic inputs, as synaptic blockade receptors resulted in a 
normalization of the increased excitability 48 hours or more after UVD. Observations in the guinea-pig one month after UVD demonstrated persistent changes in active membrane conductances [45]. These findings suggest that intrinsic excitability remains increased on the ipsilesional side, potentially in an effort to compensate long-term for the loss of excitatory vestibular afferents. .

\subsubsection{Re-organization of synaptic pathways to the MVN}

The principle of regaining balanced activity within the vestibular system is encountered not only at the level of the $\mathrm{VN}$, but also within the commissural inhibitory pathways connecting both MVN [17, 46-48]. Specifically, Bergquist and colleagues [24] demonstrated in rats that the GABA release in ipsilesional MVN neurons immediately after UVD was markedly increased. Not that this was not prevented by removal of the cerebellar flocculus on both sides, which suggests that the increase in GABA ipsilesionally was secondary to hyperactivity of contralesional commissural inhibitory neurons. As a sign of early VC, Bergquist showed that within 48 to 96 hours after UVD this excessive GABA release ipsilesionally disappeared again. Likely this was achieved by compensatory inhibition of the contralesional VN neurons. As a result, the commissural inhibitory drive mediated by contralesional VN neurons is reduced. This allows, at least partially, counteracting the lack of afferent vestibular input from the ipsilesional labyrinth. While this mechanism may be sufficient to generate an improved ipsilesional VOR for very low frequency vestibular stimuli, it will saturate by the range of accelerations used during natural head movements [7]. Other compensatory mechanisms, described below, may further improve the VOR, but usually evolve more slowly. Interestingly, static postural compensation, occurs even if the brainstem or trans-cerebellar commissures are transected at the time of UVD. Therefore, the commissural input contributes but is not a sine qua non condition for VC [32]. 


\subsubsection{Cerebellar contribution}

Vestibulo-cerebellar structures (i.e., the flocculus, nodulus, ventral uvula and the dorsal vermis) have dense reciprocal connections to the brainstem vestibular nuclei and may thus participate in VC. Early after UVD inhibitory signals from the flocculus to the VN are in fact decreased ipsilesional and increased contralesional in rats $[49,50]$. Yet, the specific role of the vestibulo-cerebellum itself remains nevertheless speculative, since some studies have reported no changes in recovery from UVD despite a deficient nodulus, flocculus or uvula $[51,52]$, while others have observed delayed (but not abolish) VC in case of preexisting cerebellar lesions affecting the nodulus, uvula [53] or flocculus [50, 54, 55].

\subsection{Mechanisms in long-term recovery}

Short-term VC is dominated by cellular mechanisms, such as increased neuronal excitability and receptor down-regulation, at the level of the $\mathrm{VN}$ and their connecting pathways. Over the long term, other mechanisms as protein synthesis, neurogenesis, synaptogenesis, synaptic remodeling and sensory substitution involving various brain regions gain importance (reviewed in [56]). Neurogenesis and astrogenesis, for example, are observed in the cat at day one after UVD, peaking at day three and continuing over at least 30 days [57]. When antimitotic drugs are applied during this period, a prominent decrease in cell proliferation and a delay in recovery of postural stability and locomotion are observed [58]. Interestingly, spontaneous nystagmus is unchanged when these processes are interrupted; suggesting that recovery of spontaneous nystagmus may rely on other plasticity mechanisms that do not require neuronal regeneration.

Reweighting of extra-vestibular sensory input likewise contributes to VC. These mechanisms resulting in sensory substitution were recently studied in the MVN of alert primates [59, 60]. After UVD, discharge rates of contralesional MVN neurons started to modulate in response to changing extra-vestibular sensory input as trunk rotations relative to 
the (stationary) head. Importantly, the increase in neck sensitivity did not lead to an enhanced cervico-ocular reflex, i.e. neck-proprioceptive driven eye movements remained negligible, as it is the case in healthy human subjects [61]. This led Sadeghi and colleagues to the hypothesis that the unmasking of neck proprioceptive input rather reflects a homeostatic mechanism allowing a continued dynamic stimulation of VN neurons after UVD and therefore supporting adaptation of vestibulo-ocular and vestibulo-spinal reflexes $[59,60]$.

With VC advancing, the brain also integrates motor efference copy signals, allowing the preprogramming of compensatory saccades at the ocular motor level [60]. This mechanism could be the basis of improved VOR-dependent gaze stability for active head movements compared to passive head movements, as observed in both humans and primates [62-64]. These changes at the level of $\mathrm{VN}$ neurons seem to parallel the behavioral improvement of the VOR for active vs. passive head movements four weeks after UVD [60], underlining their functional relevance.

\section{Recovery assessed by functional and structural brain imaging}

Demonstrations of structural and / or functional changes on brain imaging in patients that have suffered from UVD have gained increasing attention not least because of improved imaging techniques. Whereas in the acute stage of UVD metabolic changes (e.g. increased glucose uptake) dominate, adaptive processes later on may possibly be reflected by structural (volumetric) alterations of the white and gray matter.

Glucose metabolism [using fluorodeoxyglucose positron emission tomography (FDGPET)] in the multisensory vestibular cortex (including the contralateral posterior insula, hippocampus, and ipsilesional anterior cingulate gyrus) and subcortical areas (including the thalamus and the brainstem) were significantly up-regulated acutely after UVD in righthanded patients [65], reflecting the cortical correlate of peripheral vestibular tone imbalance. 
Simultaneously, glucose metabolism was down-regulated in the visual and somatosensory cortex as well as in the right temporal frontal area [66] and the superior temporal and inferior parietal lobe [65]. These down-regulations presumably reflect a cortical mechanism to suppress erroneous sensations of body motion in "secondary" vestibular cortical areas [65].

Three months after acute vestibular neuritis, voxel-based morphometry of MR-images demonstrated gray-matter volume increases within the multisensory vestibular cortical areas (insula, inferior parietal lobe, superior temporal gyrus), the cerebellum and motion-sensitive areas such as the middle temporal area MT /V5 [67]. At the same time, gray-matter volume decreases were found in the midline pontomedullary junction (i.e. the inhibitory brainstem commissural pathways interconnecting the MVN), which may either be a consequence of peripheral vestibular denervation or resulting from $\mathrm{VC}$ in order to rebalance the commissural inhibitory system. Taking into account that in this study a link between the amount of graymatter volume increase in the vestibular cortical areas and the decrease in vestibular complaints / deficits could be established, it suggests that the structural (volumetric) alterations found indeed reflect VC [67]. Except for gray-matter volume increases in the medial temporal gyrus bilaterally, gray-matter volume changes were not related to the degree of the peripheral vestibular deficit.

On the long run after acute UVD secondary to vestibular neuritis, persistent gray matter (including the bilateral MVN and the right gracile nucleus) and white matter (such as the pontine commissural vestibular fibers) volumetric increases can be demonstrated by voxel-based morphometry [68]. Functionally, the increases in the commissural system may reflect augmented vestibular crosstalk between the MVN. While gray-matter alterations were found to be independent from the side of the UVD in this study, variations in white matter signal intensities in the middle temporal gyrus were contralateral to the lesion. In the same patients, volume loss was registered in the left posterior hippocampus and the right superior temporal gyrus. Such changes could be interpreted as the result of permanently reduced 
cortical vestibular input after UVD, reflecting the limitations of VC [68]. Signs of reweighting, resulting in a shift from vestibular towards somatosensory input, were also observed at the imaging level, as suggested by increased processing of proprioceptive input in the enlarged right gracile nucleus [68] and increased importance of visual motion processing as reflected by the bilateral increase in volume of visual area V5 [68]

Taken together, imaging studies demonstrated widespread short- and long-term VC, reflected by regional metabolic changes and later on by structural alterations in diverse cortical brain areas. The amount of such gray-matter and white-matter volume changes may be used as a parameter to quantify VC in the future.

\section{The impact of $\mathrm{VC}$ on recovery}

Most patients with UVD have a good prognosis and typically return to their normal daily activities without restrictions within few weeks. However, expert opinion [69] and case series [70] suggest that about $20 \%$ fail to compensate sufficiently, suffering from chronic vestibular insufficiency. It remains unknown, why for a given peripheral deficit the functional recovery between patients varies to such an extent.

After vestibular neuritis, the hallmark disease of an acute peripheral vestibular impairment, one hypothesis regarding the great variability of clinical recovery could be the extent of vestibular nerve affection. Due to the development of manageable tools for assessing otolithic function, specifically vestibular evoked myogenic potentials [71-74], recent studies in fact show that, contrary to common presumption not only the superior but also the inferior branch of the vestibular nerve is commonly affected [75]. Further studies investigating both, the semicircular canal function by caloric and head impulse testing as well as otolithic function by vestibular evoked myogenic potentials will allow gaining a better insight on the pattern of vestibular nerve affection and on the extent of recovery. Further possible 
explanations of protracted recovery include age, the percept of vestibular symptoms including visual disturbances [76] and the previous function of the vestibular system [5, 77]. Interestingly, the overall long-term clinical outcome is independent from the amount and speed of recovery of peripheral vestibular function and age, but rather a consequence of central compensation achieved by vestibular rehabilitation [78].

\section{Conclusions}

VC triggered by acute vestibular afferent tone imbalance includes multiple parallel processes taking place at diverse locations within the brain. Whereas these mechanisms efficiently diminish static symptoms, behavioral recovery from dynamic symptoms is usually partial only, resulting in residual complaints such as blurred vision during fast head movements. Despite these limitations, the amount of $\mathrm{VC}$ achieved allows most patients to return to their normal daily activities within weeks. Why some patients fail to sufficiently compensate on the long term, however, is still poorly understood.

\section{Future perspective}

With further advances in diagnostics a more complete picture of affected peripheral vestibular structures and adaptive central mechanisms is expected. Imaging studies will likely reveal more details about the brainstem, cerebellar, and cortical areas involved in VC and their structural / functional changes. This may help answering one of the key questions unsolved, i.e. why about $20 \%$ of patients fail to centrally compensate for the unilateral vestibular deafferentation to such a degree that daily activities can be performed without restrictions, resulting in chronic vestibular insufficiency.

\section{Executive summary}

Basic principles of the vestibular system 
- The vestibular system contributes to eye movement and postural control by estimating self-motion and direction of gravity.

- The classical "three-neuron arc" originates in the vestibular end organs and projects via vestibular nerve afferents to the vestibular nuclei and further via second-order interneurons to extra-ocular motor neurons, forming the vestibulo-ocular reflexes for gaze control, and to spinal motor neurons, constituting vestibulo-spinal reflexes for balance control.

Pathophysiological changes occurring in unilateral vestibular deafferentation

- In case of an acute unilateral vestibular deafferentation (UVD) a sudden imbalance of vestibular afferent tone in the vestibular nuclei leads to profound ocular motor and postural changes and subjective complaints.

Mechanisms of vestibular compensation

- Vestibular compensation (VC) constitutes the cornerstone in restoring vestibular function after UVD, as recovery of vestibular afferents is usually insufficient.

- VC includes multiple, parallel processes taking place at diverse locations within the brain.

- The most important mechanisms in the vestibular nuclei are adapted levels of neuromodulators (as histamine and steroids) and neurotransmitters (as GABA and glycine) resulting in an increased intrinsic excitability of vestibular nucleus neurons, increased internuclear vestibular crosstalk, and neurogenesis.

- Shifts in the inhibitory control of the brainstem by the cerebellum, the replacement of vestibular by extra-vestibular (somatosensory) input and an activity-dependent reorganization of the synaptic connectivity of the vestibular pathways reflect other relevant compensatory mechanisms.

Recovery assessed by functional and structural brain imaging 
- After UVD regional metabolic changes and later on structural alterations in diverse primary and secondary vestibular brain areas are observed, likely reflecting VC.

- Gray matter and white-matter volume changes are observed in brain areas targeted as 'primary' and secondary vestibular. These changes are consistent with the principle that the brain weights visual, vestibular and somatosensory input depending on the reliability of the individual sensory signals.

\section{Clinical recovery after UVD}

- Behavioral recovery is achieved to such a degree that the majority of patients can return to their daily activities without restrictions within a few weeks.

- Why about $20 \%$ of patients fail to sufficiently compensate on the long term is still poorly understood.

\section{Figure legends}

\section{Figure 1:}

Anatomy and physiology of functional organization of the vestibular labyrinth.

Figure 1A: The bony labyrinth of the vestibular system encloses a membranous labyrinth filled with endolymph. The semicircular canals are dilated to one end forming the ampulla (left panel). The otoliths, in contrast, contain the macula, a gelatinous substance embedded with calcium carbonate crystals (right panel). The bottom of the ampulla and the macula, contain hair cells, which are innervated by bipolar sensory neurons of the vestibular nerve. When the head accelerates, inertia causes the endolymph to lag behind the motion of the membranous canal. The pressure exerted by the lag of endolymph causes bending of the 
ampulla and the macula, respectively. This, in turn displaces the hair cells, elicits a change in receptor potential and activates the vestibular nerve.

Figure 1B: Each hair cell contains 40-70 stereocilia and a single kinocilium. At rest, there is baseline release of excitatory glutamate from the hair cell synapses onto the vestibular nerve. Bending of the stereocilia toward the kinocilium leads to an influx of $\mathrm{K}^{+}$from the endolymph into the stereocilia. This causes depolarization of the hair cell and consecutively the release of transmitters, principally glutamate, resulting in an increase in firing of the afferent fibers. Conversely, bending of the stereocilia away from the kinocilium leads to hyperpolarization of the hair cell, a decrease in the release of transmitter, and a decrease in firing of the afferent fibers.

Figure 1C: Morphologically two types of hair cells can be distinguished. Flask-shaped Type I and cylindrical Type II cells. These two cell types distinguish further within their cellular properties including differences in potassium channel densities. These latter presumably explain the observed differences in the firing pattern, i.e. Type I with a regular and Type II with an irregular firing pattern.

Figure 2:

Schematic illustration (modified after [69]) of the neural basis of nystagmus elicited either by a leftward head turn in a healthy human subject (A) or by acute unilateral vestibular deafferentation (UVD) on the right side (B). In both conditions, the resulting imbalance between the medial vestibular nuclei (MVN) elicits an eye drift towards the right and a compensatory fast phase towards the left. A) A head turn leftwards excites the primary vestibular afferents of the left semi-circular canal (SCC) (thick black lines) and inhibits the primary afferents of the right SCC (dashed thin lines). MVN Type I neurons receive the 
peripheral afferent input, are excited (left side) and project to i) inhibitory Type II neurons within the contralateral MVN, ii) the contralateral (right) abducens nucleus and excite motor neurons for the right lateral rectus muscle and to iii) internuclear neurons that project to the left ocular motor nucleus via the medial longitudinal fascicle (MLF) in order to excite the left medial rectus muscle. These excitatory projections result in conjugate eye drift to the right side (slow phase, compensatory for the leftward head turn) and corrective fast phases to the left, resulting in a left-beating nystagmus. The excitation of Type II neurons in the right MVN results in inhibition of ipsilateral Type I neurons, further reducing the discharge rate of the Type I MVN neurons on the right side, as already the primary vestibular afferents originating from the right SCC are inhibitory. The decreased activity of Type I MVN neurons reduces the excitation of the contralateral abducens nucleus. At the same time inhibition of contralateral (left) Type II MVN neurons is reduced, leading to less functional inhibition of Type I neurons on the left side. Overall the discharge rates of Type I MVN neurons on the right and left are markedly distinct (onset of head motion marked with a ,"*), as graphically illustrated by the insets plotting discharge rate vs. time. B) After UVD, either due to damage to the labyrinth (1) or the vestibular nerve (2), primary afferents on the right are silenced, resulting in a dramatic drop of discharge rate in ipsilesional Type I MVN neurons and less inhibition of contralesional inhibitory Type II neurons via the commissural inhibitory pathways. This results in a severe imbalance of discharge rate at the level of the Type I MVN neurons, evoking a nystagmus identical to the one in example A. 


\section{References}

1. Kahane P, Hoffmann D, Minotti L, Berthoz A: Reappraisal of the human vestibular cortex by cortical electrical stimulation study. Ann Neurol 54(5), 615-624 (2003).

2. Tarnutzer AA, Berkowitz AL, Robinson KA, Hsieh YH, Newman-Toker DE: Does my dizzy patient have a stroke? A systematic review of bedside diagnosis in acute vestibular syndrome. CMAJ 183(9), E571-592 (2011).

3. Sirkin DW, Precht W, Courjon JH: Initial, rapid phase of recovery from unilateral vestibular lesion in rat not dependent on survival of central portion of vestibular nerve. Brain Res 302(2), 245-256 (1984).

4. Dieringer N: 'Vestibular compensation': neural plasticity and its relations to functional recovery after labyrinthine lesions in frogs and other vertebrates. Prog Neurobiol 46(2-3), 97-129 (1995).

5. Curthoys IS, Halmagyi GM: Vestibular compensation. Adv Otorhinolaryngol 55, 82110 (1999).

6. Bisdorff A, Von Brevern M, Lempert T, Newman-Toker DE: Classification of vestibular symptoms: towards an international classification of vestibular disorders. $J$ Vestib Res 19(1-2), 1-13 (2009).

7. Smith PF, Curthoys IS: Mechanisms of recovery following unilateral labyrinthectomy: a review. Brain Res Brain Res Rev 14(2), 155-180 (1989).

8. Soto E, Vega R: Neuropharmacology of vestibular system disorders. Curr Neuropharmacol 8(1), 26-40 (2010).

9. Serafin M, Khateb A, Waele C, Vidal PP, Muhlethaler M: In vitro properties of medial vestibular neurones. In: Vestibular and brain stem control of head and body movement, Shimazu H,Shinoda Y (Ed.^(Eds). Karger, Basel 111-121 (1992).

10. Matsuoka I, Domino EF: Cholinergic mechanisms in the cat vestibular system. Neuropharmacology 14(3), 201-210 (1975).

11. Spencer RF, Wang SF, Baker R: The pathways and functions of GABA in the oculomotor system. Prog Brain Res 90, 307-331 (1992).

12. Gliddon CM, Darlington CL, Smith PF: GABAergic systems in the vestibular nucleus and their contribution to vestibular compensation. Prog Neurobiol 75(1), 53-81 (2005).

13. Bergquist F, Dutia MB: Central histaminergic modulation of vestibular function - a review. Sheng Li Xue Bao 58(4), 293-304 (2006).

14. Wood CD: Antimotion sickness and antiemetic drugs. Drugs 17(6), 471-479 (1979).

15. Petrosini L, Dell'anna ME: Vestibular compensation is affected by treatment with dopamine active agents. Arch Ital Biol 131(2-3), 159-171 (1993).

16. Cransac H, Cottet-Emard JM, Pequignot JM, Peyrin L: Monoamines (norepinephrine, dopamine, serotonin) in the rat medial vestibular nucleus: endogenous levels and turnover. J Neural Transm 103(4), 391-401 (1996).

17. Precht W, Shimazu H, Markham CH: A mechanism of central compensation of vestibular function following hemilabyrinthectomy. J Neurophysiol 29(6), 996-1010 (1966).

18. Smith PF, Curthoys IS: Neuronal activity in the ipsilateral medial vestibular nucleus of the guinea pig following unilateral labyrinthectomy. Brain Res 444(2), 308-319 (1988).

19. Ris L, De Waele C, Serafin M, Vidal PP, Godaux E: Neuronal activity in the ipsilateral vestibular nucleus following unilateral labyrinthectomy in the alert guinea pig. J Neurophysiol 74(5), 2087-2099 (1995). 
20. Paterson JM, Short D, Flatman PW, Seckl JR, Aitken A, Dutia MB: Changes in protein expression in the rat medial vestibular nuclei during vestibular compensation. J Physiol 575(Pt 3), 777-788 (2006).

21. Smith PF, Curthoys IS: Neuronal activity in the contralateral medial vestibular nucleus of the guinea pig following unilateral labyrinthectomy. Brain Res 444(2), 295-307 (1988).

22. Markham $\mathrm{CH}$, Yagi T, Curthoys IS: The contribution of the contralateral labyrinth to second order vestibular neuronal activity in the cat. Brain Res 138(1), 99-109 (1977).

23. Lisberger SG, Miles FA: Role of primate medial vestibular nucleus in long-term adaptive plasticity of vestibuloocular reflex. Journal of neurophysiology 43(6), 17251745 (1980).

24. Bergquist F, Ludwig M, Dutia MB: Role of the commissural inhibitory system in vestibular compensation in the rat. J Physiol 586(Pt 18), 4441-4452 (2008).

25. Cameron SA, Dutia MB: Cellular basis of vestibular compensation: changes in intrinsic excitability of MVN neurones. Neuroreport 8(11), 2595-2599 (1997).

26. Him A, Dutia MB: Intrinsic excitability changes in vestibular nucleus neurons after unilateral deafferentation. Brain Res 908(1), 58-66 (2001).

27. Pompeiano O, Xerri C, Gianni S, Manzoni D: Central compensation of vestibular deficits. II. Influences of roll tilt on different-size lateral vestibular neurons after ipsilateral labyrinth deafferentation. J Neurophysiol 52(1), 18-38 (1984).

28. Xerri C, Gianni S, Manzoni D, Pompeiano O: Central compensation of vestibular deficits. I. Response characteristics of lateral vestibular neurons to roll tilt after ipsilateral labyrinth deafferentation. J Neurophysiol 50(2), 428-448 (1983).

29. Tolu E, Mameli O, Azzena MT, Azzena GB: Dynamic responses of vestibular cells during spinal decompensation. Physiol Behav 25(5), 637-640 (1980).

30. Lacour M, Manzoni D, Pompeiano O, Xerri C: Central compensation of vestibular deficits. III. Response characteristics of lateral vestibular neurons to roll tilt after contralateral labyrinth deafferentation. J Neurophysiol 54(4), 988-1005 (1985).

31. Matsuoka I, Fukuda N, Takaori S, Morimoto M: Responses of single neurons of the vestibular nuclei to lateral tilt and caloric stimulation in the intact and hemilabyrinthectomized cats. Acta Otolaryngol 72(3), 182-190 (1971).

32. Ris L, Capron B, De Waele C, Vidal PP, Godaux E: Dissociations between behavioural recovery and restoration of vestibular activity in the unilabyrinthectomized guinea-pig. J Physiol 500 ( Pt 2), 509-522 (1997).

33. Straka H, Vibert N, Vidal PP, Moore LE, Dutia MB: Intrinsic membrane properties of vertebrate vestibular neurons: function, development and plasticity. Prog Neurobiol 76(6), 349-392 (2005).

34. Yamanaka T, Him A, Cameron SA, Dutia MB: Rapid compensatory changes in GABA receptor efficacy in rat vestibular neurones after unilateral labyrinthectomy. $J$ Physiol 523 Pt 2, 413-424 (2000).

35. Magnusson AK, Lindstrom S, Tham R: GABA(B) receptors contribute to vestibular compensation after unilateral labyrinthectomy in pigmented rats. Exp Brain Res 134(1), 32-41 (2000).

36. Lim R, Callister RJ, Brichta AM: An increase in glycinergic quantal amplitude and frequency during early vestibular compensation in mouse. Journal of neurophysiology 103(1), 16-24 (2010).

37. Darlington CL, Smith PF: Molecular mechanisms of recovery from vestibular damage in mammals: recent advances. Prog Neurobiol 62(3), 313-325 (2000).

38. Lacour M, Tighilet B: Plastic events in the vestibular nuclei during vestibular compensation: the brain orchestration of a "deafferentation" code. Restor Neurol Neurosci 28(1), 19-35 (2010). 
39. Cameron SA, Dutia MB: Lesion-induced plasticity in rat vestibular nucleus neurones dependent on glucocorticoid receptor activation. J Physiol 518 ( Pt 1), 151-158 (1999).

40. Bergquist F, Ruthven A, Ludwig M, Dutia MB: Histaminergic and glycinergic modulation of GABA release in the vestibular nuclei of normal and labyrinthectomised rats. J Physiol 577(Pt 3), 857-868 (2006).

41. Darlington CL, Dutia MB, Smith PF: The contribution of the intrinsic excitability of vestibular nucleus neurons to recovery from vestibular damage. Eur J Neurosci 15(11), 1719-1727 (2002).

42. Nelson AB, Krispel CM, Sekirnjak C, Du Lac S: Long-lasting increases in intrinsic excitability triggered by inhibition. Neuron 40(3), 609-620 (2003).

43. Vibert N, Beraneck M, Bantikyan A, Vidal PP: Vestibular compensation modifies the sensitivity of vestibular neurones to inhibitory amino acids. Neuroreport 11(9), 19211927 (2000).

44. Guilding C, Dutia MB: Early and late changes in vestibular neuronal excitability after deafferentation. Neuroreport 16(13), 1415-1418 (2005).

45. Beraneck M, Hachemaoui M, Idoux E et al.: Long-term plasticity of ipsilesional medial vestibular nucleus neurons after unilateral labyrinthectomy. J Neurophysiol 90(1), 184-203 (2003).

46. Curthoys IS: Vestibular compensation and substitution. Curr Opin Neurol 13(1), 2730 (2000).

47. Malinvaud D, Vassias I, Reichenberger I, Rossert C, Straka H: Functional organization of vestibular commissural connections in frog. J Neurosci 30(9), 33103325 (2010).

48. Straka H, Dieringer N: Basic organization principles of the VOR: lessons from frogs. Prog Neurobiol 73(4), 259-309 (2004).

49. Ito M: Cerebellar long-term depression: characterization, signal transduction, and functional roles. Physiol Rev 81(3), 1143-1195 (2001).

50. Kitahara T, Takeda N, Kiyama H, Kubo T: Molecular mechanisms of vestibular compensation in the central vestibular system--review. Acta Otolaryngol Suppl 539, 19-27 (1998).

51. Schaefer KP, Meyer DL, Wilhelms G: Somatosensory and cerebellar influences on compensation of labyrinthine lesions. Prog Brain Res 50, 591-598 (1979).

52. Haddad GM, Friendlich AR, Robinson DA: Compensation of nystagmus after VIIIth nerve lesions in vestibulo-cerebellectomized cats. Brain Res 135(1), 192-196 (1977).

53. Igarashi M, Ishikawa K: Post-labyrinthectomy balance compensation with preplacement of cerebellar vermis lesion. Acta Otolaryngol 99(3-4), 452-458 (1985).

54. Courjon JH, Flandrin JM, Jeannerod M, Schmid R: The role of the flocculus in vestibular compensation after hemilabyrinthectomy. Brain Res 239(1), 251-257 (1982).

55. Johnston AR, Seckl JR, Dutia MB: Role of the flocculus in mediating vestibular nucleus neuron plasticity during vestibular compensation in the rat. $J$ Physiol $545(\mathrm{Pt}$ 3), 903-911 (2002).

56. Dieringer N: Activity-related postlesional vestibular reorganization. Ann N Y Acad Sci 1004, 50-60 (2003).

57. Tighilet B, Brezun JM, Sylvie GD, Gaubert C, Lacour M: New neurons in the vestibular nuclei complex after unilateral vestibular neurectomy in the adult cat. Eur $J$ Neurosci 25(1), 47-58 (2007).

58. Dutheil S, Brezun JM, Leonard J, Lacour M, Tighilet B: Neurogenesis and astrogenesis contribution to recovery of vestibular functions in the adult cat following 
unilateral vestibular neurectomy: cellular and behavioral evidence. Neuroscience 164(4), 1444-1456 (2009).

59. Sadeghi SG, Minor LB, Cullen KE: Multimodal integration after unilateral labyrinthine lesion: single vestibular nuclei neuron responses and implications for postural compensation. J Neurophysiol 105(2), 661-673 (2011).

60. Sadeghi SG, Minor LB, Cullen KE: Neural correlates of motor learning in the vestibulo-ocular reflex: dynamic regulation of multimodal integration in the macaque vestibular system. J Neurosci 30(30), 10158-10168 (2010).

61. Roy JE, Cullen KE: Vestibuloocular reflex signal modulation during voluntary and passive head movements. J Neurophysiol 87(5), 2337-2357 (2002).

62. Dichgans J, Bizzi E, Morasso P, Tagliasco V: Mechanisms underlying recovery of eye-head coordination following bilateral labyrinthectomy in monkeys. Exp Brain Res 18(5), 548-562 (1973).

63. Newlands SD, Hesse SV, Haque A, Angelaki DE: Head unrestrained horizontal gaze shifts after unilateral labyrinthectomy in the rhesus monkey. Exp Brain Res 140(1), 25-33 (2001).

64. Della Santina CC, Cremer PD, Carey JP, Minor LB: Comparison of head thrust test with head autorotation test reveals that the vestibulo-ocular reflex is enhanced during voluntary head movements. Arch Otolaryngol Head Neck Surg 128(9), 1044-1054 (2002).

65. Bense $\mathrm{S}$, Bartenstein $\mathrm{P}$, Lochmann $\mathrm{M}$, Schlindwein $\mathrm{P}$, Brandt $\mathrm{T}$, Dieterich $\mathrm{M}$ : Metabolic changes in vestibular and visual cortices in acute vestibular neuritis. Ann Neurol 56(5), 624-630 (2004).

66. Alessandrini M, Napolitano B, Bruno E, Belcastro L, Ottaviani F, Schillaci O: Cerebral plasticity in acute vestibular deficit. Eur Arch Otorhinolaryngol 266(10), 1547-1551 (2009).

67. Helmchen C, Klinkenstein J, Machner B, Rambold H, Mohr C, Sander T: Structural changes in the human brain following vestibular neuritis indicate central vestibular compensation. Ann N Y Acad Sci 1164, 104-115 (2009).

68. $\mathrm{Zu}$ Eulenburg P, Stoeter P, Dieterich M: Voxel-based morphometry depicts central compensation after vestibular neuritis. Ann Neurol 68(2), 241-249 (2010).

69. Halmagyi GM, Weber KP, Curthoys IS: Vestibular function after acute vestibular neuritis. Restor Neurol Neurosci 28(1), 37-46 (2010).

70. Reid CB, Eisenberg R, Halmagyi GM, Fagan PA: The outcome of vestibular nerve section for intractable vertigo: the patient's point of view. Laryngoscope 106(12 Pt 1), 1553-1556 (1996).

71. Jacobson GP, Mccaslin DL, Piker EG, Gruenwald J, Grantham SL, Tegel L: Patterns of abnormality in cVEMP, oVEMP, and caloric tests may provide topological information about vestibular impairment. $J$ Am Acad Audiol 22(9), 601-611 (2011).

72. Kim HA, Hong JH, Lee $\mathrm{H}$ et al.: Otolith dysfunction in vestibular neuritis: recovery pattern and a predictor of symptom recovery. Neurology 70(6), 449-453 (2008).

73. Nola G, Guastini L, Crippa B, Deiana M, Mora R, Ralli G: Vestibular evoked myogenic potential in vestibular neuritis. Eur Arch Otorhinolaryngol 268(11), 16711677 (2011).

74. Viciana D, Lopez-Escamez JA: Vestibular evoked myogenic potentials and healthrelated quality of life in patients with vestibular neuritis. Otology \& neurotology : official publication of the American Otological Society, American Neurotology Society [and] European Academy of Otology and Neurotology 31(6), 954-958 (2010).

75. Kim JS, Kim HJ: Inferior vestibular neuritis. Journal of neurology 259(8), 1553-1560 (2012). 
76. Guinand N, Pijnenburg M, Janssen M, Kingma H: Visual acuity while walking and oscillopsia severity in healthy subjects and patients with unilateral and bilateral vestibular function loss. Archives of otolaryngology--head \& neck surgery 138(3), 301-306 (2012).

77. Nuti D, Mandala M, Broman AT, Zee DS: Acute vestibular neuritis: prognosis based upon bedside clinical tests (thrusts and heaves). Ann N Y Acad Sci 1039, 359-367 (2005).

78. Strupp M, Arbusow V, Maag KP, Gall C, Brandt T: Vestibular exercises improve central vestibulospinal compensation after vestibular neuritis. Neurology 51(3), 838844 (1998). 


\section{Reference annotations:}

** Sadeghi SG, Minor LB, Cullen KE: Neural correlates of motor learning in the vestibuloocular reflex: dynamic regulation of multimodal integration in the macaque vestibular system. J Neurosci 30(30), 10158-10168 (2010).

REASON: This study nicely demonstrates sensory substitution after UVD at the level of the vestibular nuclei.

* Bergquist F, Ludwig M, Dutia MB: Role of the commissural inhibitory system in vestibular compensation in the rat. J Physiol 586(Pt 18), 4441-4452 (2008).

REASON: This study highlights the key role of the commissural inhibitory system in VC.

* Dutheil S, Brezun JM, Leonard J, Lacour M, Tighilet B: Neurogenesis and astrogenesis contribution to recovery of vestibular functions in the adult cat following unilateral vestibular neurectomy: cellular and behavioral evidence. Neuroscience 164(4), 1444-1456 (2009).

REASON: The authors demonstrate that neurogenesis evolves during $\mathrm{VC}$ and contributes to the behavioral recovery after UVD.

* Johnston AR, Seckl JR, Dutia MB: Role of the flocculus in mediating vestibular nucleus neuron plasticity during vestibular compensation in the rat. J Physiol 545(Pt 3), 903-911 (2002).

REASON: This study showed that the increase in intrinsic excitability in MVN neurons during vestibular compensation is cerebellum dependent. 
* Bense S, Bartenstein P, Lochmann M, Schlindwein P, Brandt T, Dieterich M: Metabolic changes in vestibular and visual cortices in acute vestibular neuritis. Ann Neurol 56(5), 624630 (2004).

REASON: Area-specific acute metabolic changes after UVD are demonstrated, showing increased activity in vestibular and decreased activity in visual and somatosensory areas.

** Zu Eulenburg P, Stoeter P, Dieterich M: Voxel-based morphometry depicts central compensation after vestibular neuritis. Ann Neurol 68(2), 241-249 (2010).

REASON: This study highlights the structural changes within vestibular, visual and somatosensory areas related to VC after UVD.

* Strupp M, Arbusow V, Maag KP, Gall C, Brandt T: Vestibular exercises improve central vestibulospinal compensation after vestibular neuritis. Neurology 51(3), 838-844 (1998).

REASON: Strupp and colleagures report on the impact of vestibular physiotherapy on vestibulospinal compensation, demonstrating its usefulness in patients with acute peripheral vestibular lesions. 
A
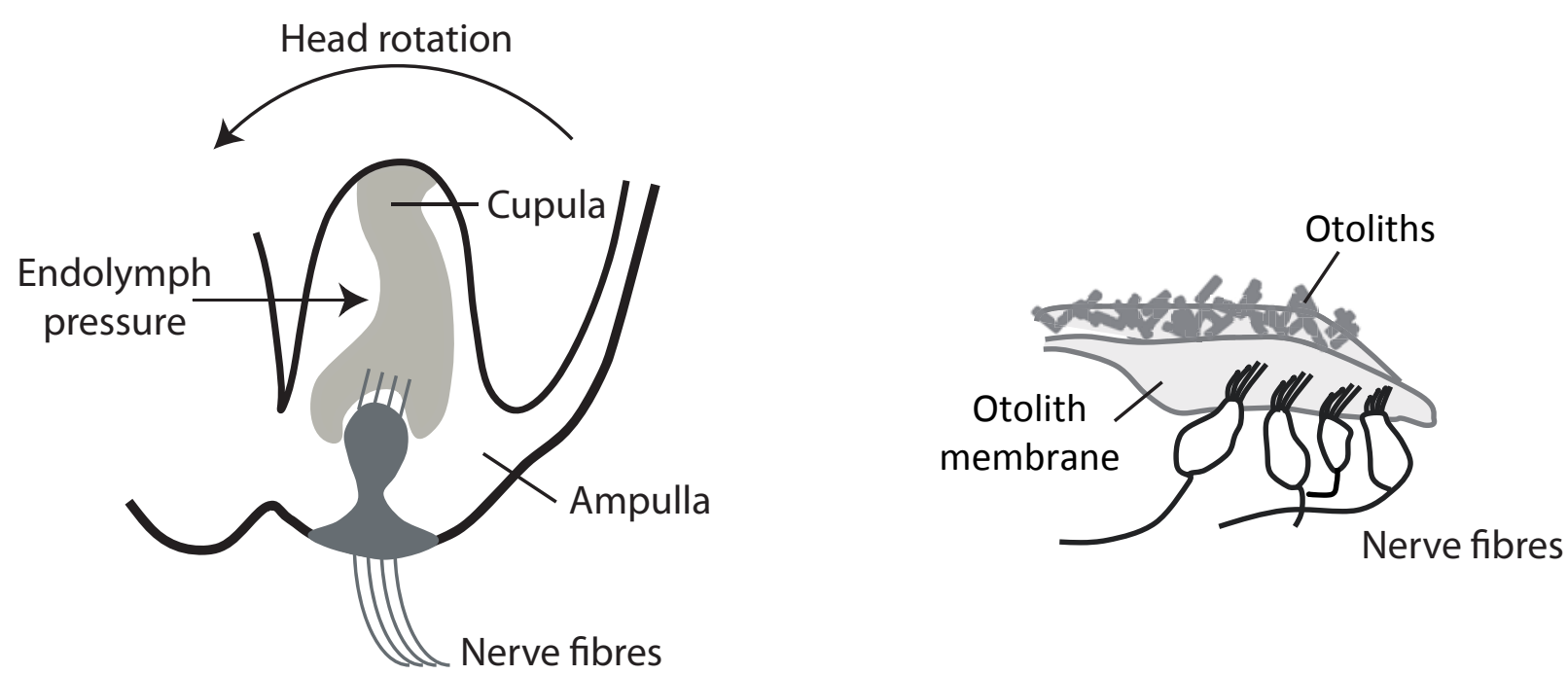

B hair cell

hair cell

depolarization hyperpolarization

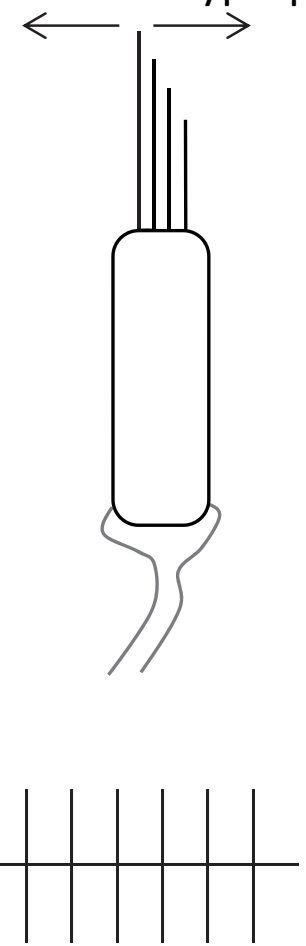

Type I cell regular afferent
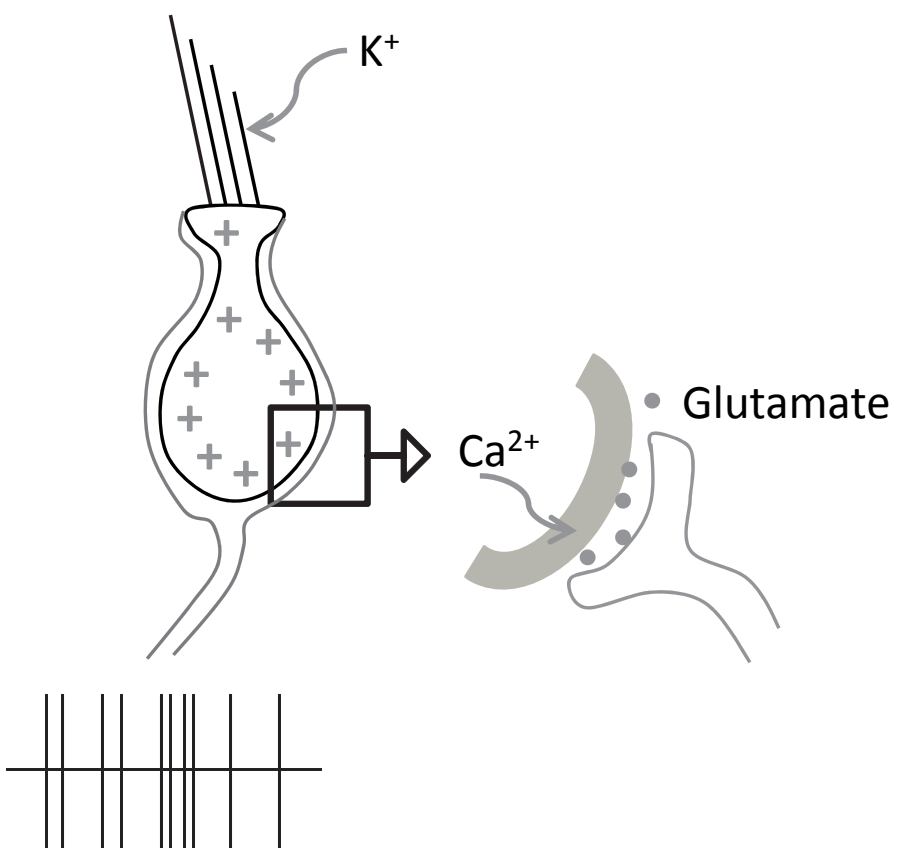

Type II cell irregular afferents 
